Journal of Applied Fluid Mechanics, Vol. 9, Special Issue 1, pp. 39-48, 2016. Selected papers from the $7^{\text {th }}$ International Exergy, Energy

and Environment Symposium, IEEE7-2015

Available online at www.jafmonline.net, ISSN 1735-3572, EISSN 1735-3645.

DOI: 10.36884 /jafm.9.SI1.25822

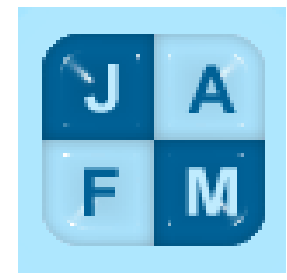

\title{
Liquid Film Thickness: Study and Influence over Aqueous Foam Flow
}

\author{
R. Chovet and F. Aloui ${ }^{\dagger}$ \\ University of Valenciennes and Hainaut-Cambresis (UVHC), LAMIH CNRS UMR 8201, Department of \\ Mechanics \\ Campus Mont Houy, F-59313 Valenciennes Cedex 9, France \\ †Corresponding Author Email: Fethi.Aloui@uni-valenciennes.fr
}

(Received October 20, 2015; accepted December 10, 2015)

\begin{abstract}
Nowdays, we face important energy challenges. These ones are making scientist all over the world reconsider the way they look into problem and find innovative solutions to improve industrial processes efficiency. One of many original ideas is the use of "not-regular" fluids over regular applications. Aqueous Foam Flow present several unusual rheological properties when put inside a horizontal channel: low density, visco-elasto-plastic behaviour, and high wall shear stress. These ones give this type of fluid interesting capacities and uses: Assisted oil extraction, heat exchange, lubrication. In this study we undertake the causes of these interesting properties, which are directly related to the liquid slip-layer located between the flowing bubbles and the walls. For different velocities $(2 \mathrm{~cm} / \mathrm{s}, 4 \mathrm{~cm} / \mathrm{s}$ and $6 \mathrm{~cm} / \mathrm{s}$ ) and void fractions (from $55 \%$ to $85 \%$ ) we will study the influence of the liquid film thickness over the wall shear stress, using innovative measurement techniques: conductimetry and polarographic methods. An interesting relationship is seen between the limit diffusion current, required to accurately utilize the polarographic method, the wall shear stress and the liquid film thickness. The bubbles passage over the walls generates an oscillation of the slip-layer thickness which directly affects the polarographic results. However, as we increase the foams velocity this influence diminish and the wall shear stress calculations are more accurate.
\end{abstract}

Keywords: Foam flow; wall shear stress; PIV; Energy; Conductimetry method; Void fraction; Efficiency.

NOMENCLATURE

$\begin{array}{ll}d & : \text { diameter } \\ e & : \text { unit } \\ g & \text { : gravity } \\ h & : \text { water column height } \\ L & : \text { channel's lenth } \\ P & : \text { pressure } \\ Q & : \text { flow } \\ u & : \text { axial velocity component } \\ v & : \text { fluid velocity } \\ x, y, z & : \text { coordinates } \\ & \\ \text { Greek letters } \\ \beta & : \text { foam quality } \\ \Delta & : \text { diffence } \\ \mu & : \text { viscosity (Pa.s) }\end{array}$

\section{Introduction}

What defines foam flow? Why does it generate high pressure losses? Different works, coming most of them with similar answers, have discussed this item.
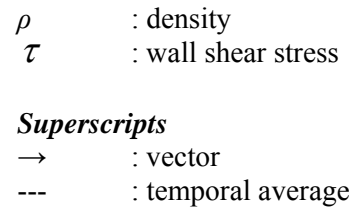

$\begin{array}{ll}\text { Subscripts } \\ h & : \text { hydraulic } \\ x & : \text { x axis } \\ a & : \text { atmospheric } \\ g & : \text { gas } \\ l & : \text { liquid }\end{array}$

The main objective of this experimental study has to do with this question; we approach it by studying the influence that the void fraction has over the liquid slip-layer generated at the channel's walls, the pressure losses and the wall shear stress. 
Foams are formed by trapping pockets of air inside a liquid or a solid. They may contain more or less liquid. Dry foams, for instance, have lesser liquid in thin films. The bubbles form polyhedral cells, which are not flat. The films meet in lines (edges of the polyhedral), and the lines meet at vertices. In tow dimensions, the dry foam consists of polygonal cells (Fig. 1.a). The liquid is mainly to be found in Plateau borders, which are channels of finite width, replacing the lines in the dry foam. As the fraction of liquid increases, the swelling of the plateau borders eventually leads to the extreme limit of a wet foam. The bubbles recover a spherical form and any further increase of liquid will make them to fall apart. Then the foam losses its rigidity and it's replaced by a bubbly liquid. For a two dimensional case the bubbles become circular at the stability film (Fig. 1.b). The liquid excess is pushed towards the walls creating a slipping-layer over which bubbles slide (Weaire and Hutzler, 1999).

Foams may be classified as dry or wet accordingly to its liquid content, which may be represented by the gas volume fraction. Engineers call this one "Foam quality"; it can be represented by the equation:

$\beta=\frac{Q_{g}}{Q_{l}+Q_{g}}$

where Qg is the gas flow and Q1 is the liquid one.

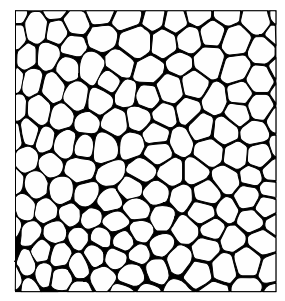

(a)

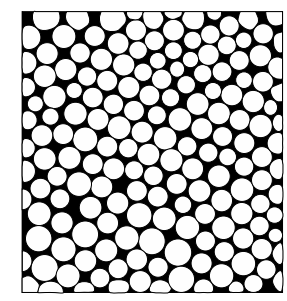

(b)
Fig. 1. Dry and wet limit (Stevenson, 2012).

It ranges from less than $1 \%$ to about $30 \%$. At each extreme (the dry and the wet limits) the bubbles come together to form a structure which resembles one of the classic idealized paradigms of nature's morphology: The division of cells in the dry limit, and the close packing of spheres in the wet limit.

It is important to indicate the sizing and scales considered in foams (Fig 2). The smallest length-scales are that of the surfactant molecules, typically in the order of nanometers. The forces of repulsion (often expressed as a disjoining pressure) determine the thickness of the film in the range 5 $\mathrm{nm}$ to $10 \mathrm{~nm}$ in relative dry foam. There is a medium scale usually applied by physicists to the relation between bubbles. The bubbles diameter is widely variable, but is in the order of a few millimeters. In an engineering approach, the air and liquid phases are taken as a continuum model. It is normally studied as a foam flow (Stevenson, 2012).

Treated as a macroscopic system, foam elements undergo no significant thermal fluctuation, by means of which it might explore alternatives to the

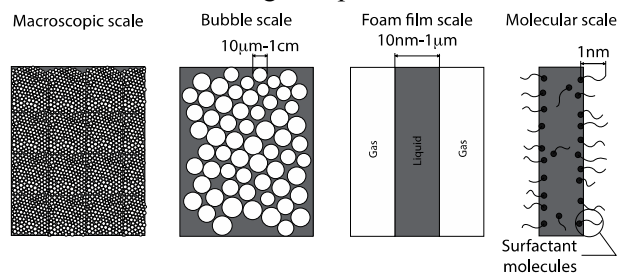

Fig. 2. Characteristic length of the foam.

local minimum of energy in which it finds itself (Weaire and Hutzler, 1999). It is not considered to be in a metastable state, since it continually evolves according to the coarsening process, air exchange between neighbor bubbles (Durian et al. 1990). However, this is a very slow process. The foam therefore stays very close to a true equilibrium except where a local topological change may take place.

When the topological structure of the foam is altered, it may be brought to a configuration in which there is a violation of the Plateau's rule by the introduction of a "forbidden" vertex. This configuration dissociates rapidly and a new structure is formed. For 2D flow, the possibilities are rather simple: The so-called $\mathrm{T} 1$ process eliminates the fourfold vertex and forms two threefold ones, while the $\mathrm{T} 2$ process is associated to the disappearance of a bubble, also known as coalescence. Both processes are sudden events in which the surface energy of the foam drops abruptly and the energy loss is dissipated as heat (Hutzler et al. 2008). The bubble rearrangement leads to local shear flow of the liquid inside the foam films, resulting in dissipation of energy and shear-rate dependent contribution to the macroscopic stress.

Foam has unusual rheology properties: they present low densities and an important interfacial surface. They are mainly composed of gas. But under low applied stress, foam behaves as a solid; hence shaving foam adheres to the shaver's face, the weak force of gravity being incapable of letting it flow. This results from an elastic shear modulus, as occurs in isotropic solid materials. They can behave only as an elastic solid or a plastic one. Beyond a certain yield stress foam flows, as topological changes are promoted indefinitely (Denkov et al. 2012).

Foam flow has become a mayor study subject for industries around the world. For exampler, poor a bootle of beer. One by one, bubbles of gas are nucleated, rise and crowd together at the surface. A foam is quickly formed. Most of the liquid drains away; leaving the bubbles packed closely together in the form of elegant polyhedral cells. This gradual change is punctuated by sudden local rearrangements. Drink it in time, and you can feel the foam is not q liauid. Paradoxically, it is a soft solid, creamy in texture if the bubbles are small.

It is indispensable to better understand its main characteristics especially the knowledge of the rheological behaviour and of the foam stability. 
Most of the studies carried out focus on the geometry and topology of stationary foam and not on the flow itself, due to its complexity (Weaire and Hutzler, 1999; Stevenson, 2012). However, there have been important experimental works that undertook the task of examinate the flow of foams (Blondin and Doubliez, 2002; Aloui and Madani, 2007; Aloui and Madani, 2008). Looking at the high interest shown for foam flow along the industries, it is indispensable to achieve the capacity to understand and evaluate all the variables concerning the hydraulic behaviour.

\section{Experimental Facility}

\subsection{Setup}

The experimental facility was designed to handle the different requirements of foam flow. It has a geometry capable of making studies for horizontal foam flows. It presents plane walls making it suitable for the experimental measurements undertaken. Thus, the plane walls allowed the easy placement of polarographic and conductimetry probes along its surface. The test duct has a length of approximately $3.2 \mathrm{~m}$. It is made out of Plexiglas with a $21 \times 21 \mathrm{~mm}^{2}$ square section. The channel is located on permanent supports that assure its horizontality. The exit of the test section is open to the atmosphere (Fig. 3).

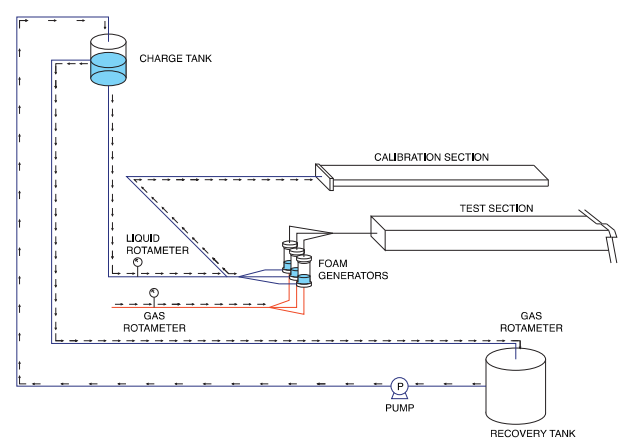

Fig. 3. Schematic sketch of the experimental facility.

The foam is generated by injecting pressured air, through a permeable media inside cylindrical containers filled with liquid, which act as foam generator. The water contains surfactants that lower the surface tension of the liquid and allow the formation of bubbles (Fig. 4). The presence of three generators, parallels and independents, allow us to modify the foam flow and bulk velocity in three cases (one generator for $2 \mathrm{~cm} / \mathrm{s}$, two for $4 \mathrm{~cm} / \mathrm{s}$, and three for $6 \mathrm{~cm} / \mathrm{s}$ ) without affecting the foam structure.

A way to evaluate horizontal foam flow inside a channel is through its global velocity for a foam quality of $70 \%$ three different regimes, can be observed. They result from the interpolation of the velocity and, measured at the ducts wall, over a straight section. The three different flow regimes are (Blondin and Doubliez, 2002):

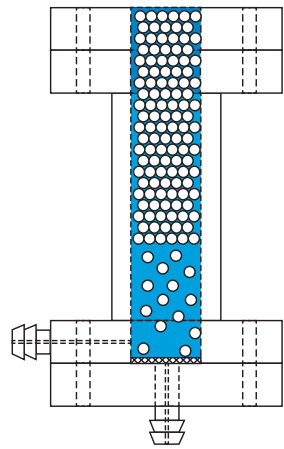

Fig. 4. Diagramme of the foam generator.

* One-dimensional flow: for this regime, the flow behaves as a whole, it moves like a block or a piston. The velocity vectors have one uniform axial component in the flow direction.

$\vec{u}=u \cdot \vec{e}_{x}$

* Two-dimensional flow: it is obtained when the established flow has an axial velocity component that only depends of the y coordinate.

$\vec{u}=u(y) \cdot \vec{e}_{x}$

* Three-dimensional flow: this one is obtained when the established flow velocity vector presents an axial component that depends on the $\mathrm{z}$ and $\mathrm{y}$ coordinates.

$\vec{u}=u(y, z) \cdot \vec{e}_{x}$

\subsection{Measurements techniques}

The static pressure along the test section is measured by pressure outlets located at the bottom of the channel, at the middle vertical plane. These pressure outlets are attached through silicon hoses, to a series of manifold tubes placed over a scaled plate (Fig. 5). The misreading of the manifold pipes is estimated at a maximum $1 \mathrm{~mm}$ water column. There are 8 pressure outlets along the channel length.

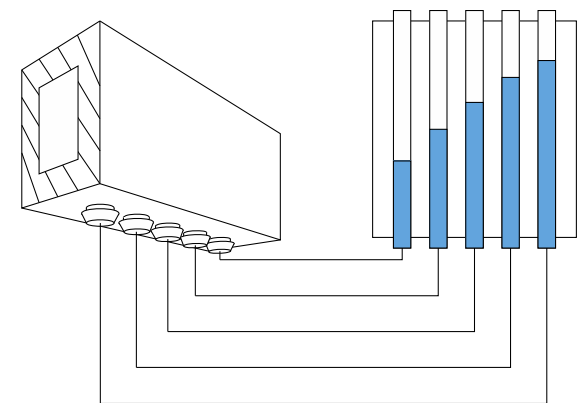

Fig. 5. Schematic sketch of the pressure outlets arrangement.

The measured static pressure value at the wall is determined using the hydrostatic equation:

$P=P_{a}+\rho g \Delta h$ 
where $\mathrm{Pa}$ is the atmospheric pressure, $\Delta h$ the height difference between the water column height measured at the pressure outlet and the one measured for the atmospheric pressure, $\rho$ the manometric liquid density and $g$ the gravity acceleration.

In two phase foam flow, there is some methods to measure the wall-slip layer close to the walls. These techniques go from the micro-needles, capable of detecting the gas phase, to the $\mathrm{x}$-ray absorption. The foam flow carried a liquid layer at the walls induced by the drainage and internal forces. To measure it, we used in our case the conductimetry method. It can measure the electric resistance of the liquid film between the foam bubbles flowing over the wallsand the probes which are mounted flush in these walls. This method can be applied to any electric conductive liquid flowing over a non-conductive surface. It is simple to use being a non-intrusive method; the probes don't influence the foam flow pattern.

To accurately measure different liquid thicknesses; two measurement blocks were set, each with a different probe size. The small one was used for the wall-slip layer at the top and the sides of the duct, and the large one for the thickest liquid film at the bottom. The electrodes are powered in each block with an alternative tension of sinusoidal form $( \pm 15$ $\mathrm{V})$. The frequency is high enough to neglect the polarization phenomena (between 50 and $100 \mathrm{kHz}$ ). Therefore, quantifying the micrometric liquid film can be considered. A simplified diagram of the conductimetry method is given in Fig. 6 .

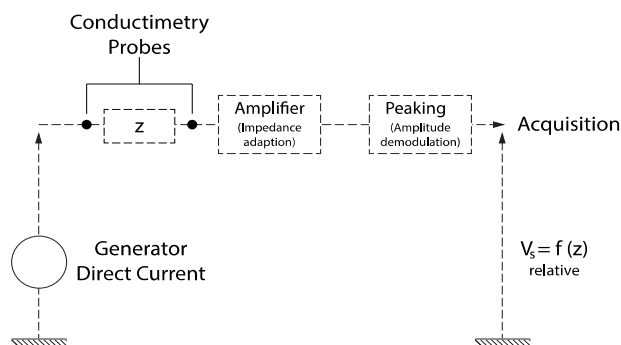

Fig. 6. Simplified diagram of the conductimetry method.

The two blocks used to measure the wall liquid film thickness include the following elements:

* The first one (block A, where probes are the the larger ones) measures the thickness of the bottom underlying liquid film. It is equipped with two electrodes. Each one has $40 \mathrm{~mm} 2$ of surface, and they are $6 \mathrm{~mm}$ away from each other. They are made out of platinum.

* The second one (block B) is used to measure the smaller film liquid layers, at lateral sides and top of the channel. It also carries two probes constituting one conductimetric probe. These two probes have each one a diameter of $0.25 \mathrm{~mm}$ and they are separated by a distance of about $6 \mu \mathrm{m}$. They are made out of platinum, and they were placed in a way that allows obtaining the thickness profile along the whole wide or hight of the used channel. To achieve it, the block is graduated from 0 to $180^{\circ}$ (Fig 7) and can turn around its center. The value $\alpha=0^{\circ}$ corresponds to the highest position which is $19.5 \mathrm{~mm}$ from the bottom of the channel, and $\alpha=180^{\circ}$ is the lowest position situated at $1.5 \mathrm{~mm}$ from the channel bottom.



Fig. 7. Positioning of the conductimetry probes over the exchangeable blocks.

The velocity measurements were done using a standard Particle Image Velocimetry based on a TSI Power view TM system, including a light sheets provided by a double-pulsed Nd-YAG laser operating at $532 \mathrm{~nm}$ (green), with a $7.25 \mathrm{~Hz}$ frequency, and high resolution. It also used a PowerviewTM 4MP camera, a synchronizer (TYPE) and Insight $($ as the software for the acquisition, processing and post processing. The maximum energy of each pulse was $200 \mathrm{~mJ}$ for $8 \mathrm{~ns}$.

The PIV method is a non-intrusive optic technique, able of measuring the displacement of particles in a plane. It is done by the comparison between two instantaneous position fields of particles in the flow. It is possible to calculate mean velocity fields and instantaneous vorticity by this particle displacement. A diagram of this method is represented in Fig. 8.

In foam flow, the gas/liquid interphase is darker than the rest of the flow, and the bubbles contour assigns the whole flow movement (not the movement of each bubble). Therefore, the use of particles is not necessary to track the flow. The close packing of bubbles keeps them together along the channels length, avoiding the error that bubbles displacement might generate. The measures were made over the channel lateral wall. Due to the opacity of the foam flow, and the reflections over the bubbles it is impossible to see through it, and obtain the internal characteristics.

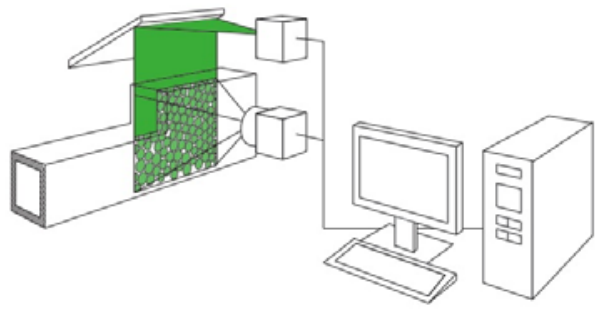

Fig. 8. Overview of the PIV system. 
R. Chovet et al. / JAFM, Vol. 9, Special Issue 1, pp. 39-48, 2016.

\section{Results and discussions}

The experimental results obtained from the pressure outlets, the conductimetry and PIV method are present in the form of pressure losses, liquid slip-layer thickness, and velocity fields and profiles, respectively.

\subsection{Static pressure losses and gradient}

The pressure distribution generated by foam flowing along the channel is one of the most interesting characteristics of this complex fluid. The static pressure losses inside the channel are represented in Fig. 11. In all cases, the pressure varies linearly all through the channel. Regardless of the change in the foam quality, the difference between one static pressure to the other is relatively small

The longitudinal pressure gradient was obtained from the static measurements. They are shown in Fig. 9 as a function of the foam quality. Case III ( 6 $\mathrm{cm} / \mathrm{s}$ ) presents a linear pressure gradient. However, for cases II and I there is an influence of the foam quality over the value of the gradients. They show that wetter foams generate higher-pressure losses and that the changes, in the linearity of the pressure gradients, can be a result of the change in the foam velocity profiles and the film liquid thickness at the walls. Therefore, a deeper study was carried to understand the real causes of this behavior.



Fig. 9. Static pressure losses along the square channel without flow restrictions: (a) case I, (b) case II and (c) case III.

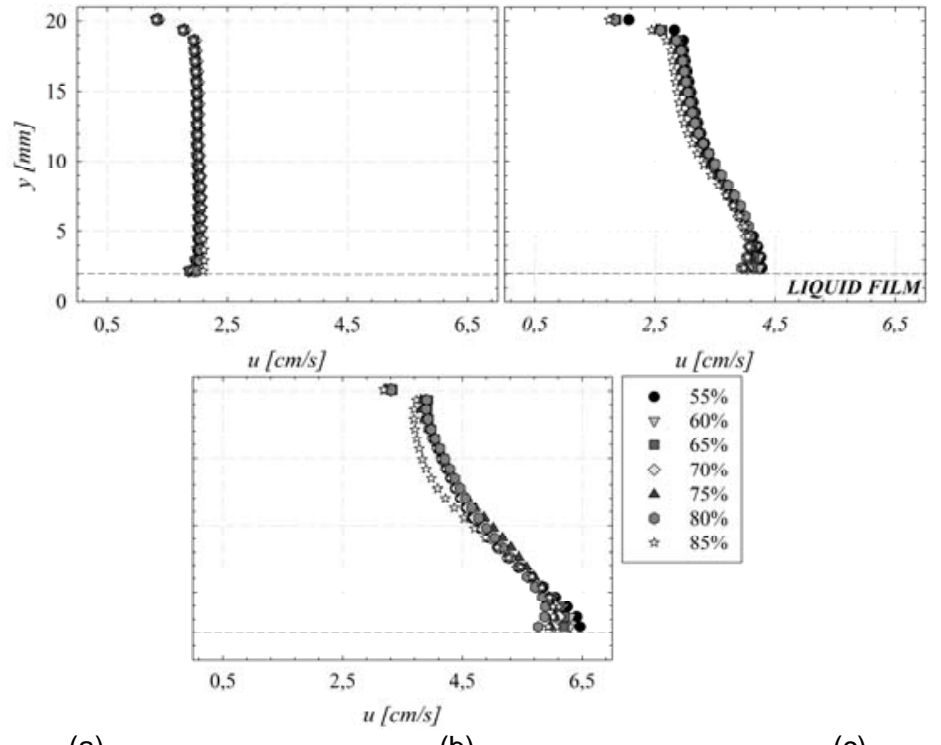

(a)

(b)

(c)

Fig. 10. Average axial velocity profiles at the lateral wall of the foam flowing through a straight channel with different qualities for: case I (a), case II (b) and case III (c). 


\subsection{Velocity profiles}

Indicated in previous works (Blondin and Doubliez 2002; Chovet et al. 2013; Aloui and Madani 2007; Aloui and Madani 2008); the foam flowing through channels may be studied under three different regimes, as a function of the axial velocity. This characteristic can be measured by the PIV method. The axial velocity profiles for all cases and qualities, are presented as average velocity values, taken over 500 images (Fig. 10).

For the case I, where the foam behaves as a block (or piston), there is small influence of the void fraction, in comparison with the other cases. Due to thinner plateau borders, and weaker internal forces, dryer foams tend to shear easier than wet ones. However, as the liquid flow increases so do the thickness and velocity of the layer at the bottom of the channel. Therefore, for cases II and III; wetter foams have a higher velocity at the bottom of the duct. As the gas fraction increases, the liquid slip layer velocity decreases, arriving to the driest point, where shear occurs and the foam at the bottom deforms easier. The higher the mean velocity is, the higher the influence of the liquid film.

\subsection{Liquid slip-layer thickness}

Aqueous foam flow consists of a mixture of water and air. Therefore, the gravitational force plays a major impact over its behavior and more specifically its drainage. Drainage is the passage of liquid through foam, under the effects of gravity, the liquid inside the foam flows downward, and the gas bubbles are displaced upwards in the channel section. The conductimetry method allowed defining the liquid slip layer experimental thickness value over three of the four walls of the duct: bottom, one lateral, and top.

Fig 11 represents the mean values of the liquid slip layer for all cases and qualities at the bottom. They were obtained with the use of the conductimetry block A, located at the middle of the test section. They show that the change of the foam velocity does not make an impact over the mean value of the liquid slip layer; there are no major deviations between the thicknesses of the three cases. However, as we alter the foam quality the value of liquid film changes importantly. Therefore, for wetter foams the slip layer is thicker than for dryer ones. Previous works have shown that there is no mayor thickness evolution along the channel's length, meaning that the liquid quantity lost, due to the gravitational drainage over horizontal flowing foam inside a square channel, is small.

The lateral wall measurements were made with the conductimetry block B. By turning it around its center, it allows the acquisition of the liquid film thicknesses over the lateral wall (from $y=1.5 \mathrm{~mm}$ to $19.5 \mathrm{~mm}$ ). Fig 12 represents an example of the instantaneous conductimetry signals evolution over one of the two lateral walls, for all qualities at a velocity of $2 \mathrm{~cm} / \mathrm{s}$. For each condition, we represent

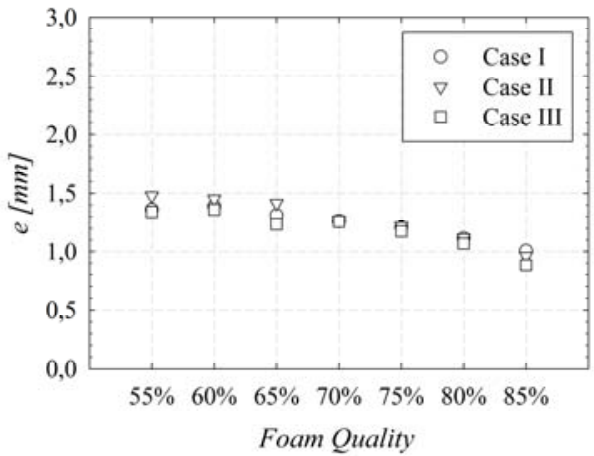

Fig. 11. Mean liquid film thickness at the bottom of the channel for all cases and qualities.

four of the thirteen instantaneous signals available. The rest of them were used to obtain the mean value evolution. Over the lateral wall, the probes were located near the top $(\mathrm{y}=19.5 \mathrm{~mm})$, at the middle (y $=16.9 \mathrm{~mm}$ and $4.1 \mathrm{~mm})$ and the bottom $(\mathrm{y}=1.5$ $\mathrm{mm}$ ) of the channel. These four ones allow visualizing partially the fluctuations evolution over the lateral wall. For all conditions, the instantaneous signal obtained at the conductimetry probe, show important fluctuations all over the lateral wall. Previous works have made an emphasis over this phenomenon and the causes of it (Tisné et al. 2004).

The mean values, of the conductimetry signal over the lateral wall, are represented in Fig. 13 for all cases and qualities. They allow seeing the evolution of the thickness and the influence of the drainage over this one. The liquid iniside the foam, due to the gravity force, tends to the bottom of the channel. Therefore at this region the film is thicker.The sliplayer thickness diminishes almost $40 \mu \mathrm{m}$ along the whole wall. For all three cases the minimum value is of $30 \mu \mathrm{m}$ at the summit of the conduit and $70 \mu \mathrm{m}$ at the bottom. There is no big difference between foam velocities or qualities.

Finally, Fig. 14 presents an example of the instantaneous evolution of the slip-layer thickness over the top wall for all foam qualities and a $2 \mathrm{~cm} / \mathrm{s}$ velocity. These values were also obtained with the conductimetry block B, which has smaller probes and precision. It was turned around its center and allowed the measure over the whole wall. We present three instantaneous signals near the lateral walls $(z=-9 \mathrm{~mm}$ and $9 \mathrm{~mm})$ and at the center of the channel $(z=0 \mathrm{~mm})$. These ones are more than enough to obtain the partial view of the evolution along the wall. At the top of the channel the slip layer thickness fluctuation goes from $2 \mu \mathrm{m}$ to 40 $\mu \mathrm{m}$. The mean liquid film thicknesses may be obtained from the instantaneous values of the conductimetry signal over the top wall. These ones are shown in Fig. 15, for all qualities and velocities. As expected from the instantaneous fluctuations, on the three cases, the mean liquid film thickness over the wall presents similar values for all the conditions. It tends to $30 \mu \mathrm{m}$. 
R. Chovet et al. / JAFM, Vol. 9, Special Issue 1, pp. 39-48, 2016.

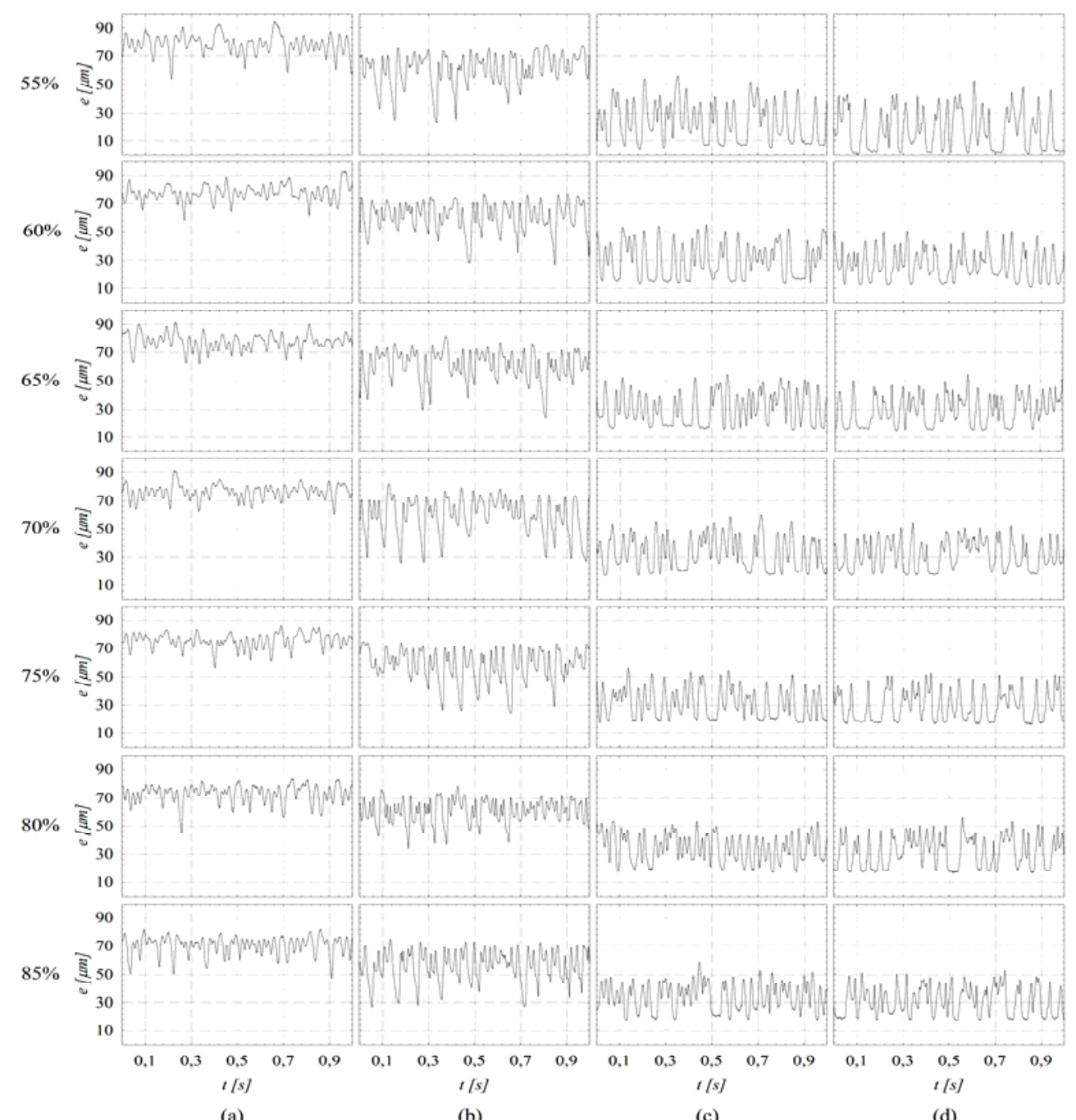

Fig. 12. Conductimetry signal over the lateral wall for a foam velocity of $2 \mathrm{~cm} / \mathrm{s}$ at: (a) $y=1.5 \mathrm{~mm}$, (b) $\mathrm{y}=4.1 \mathrm{~mm}$, (c) $\mathrm{y}=16.9 \mathrm{~mm}$ and (d) $\mathrm{y}=19.5 \mathrm{~mm}$ for all foam qualities.

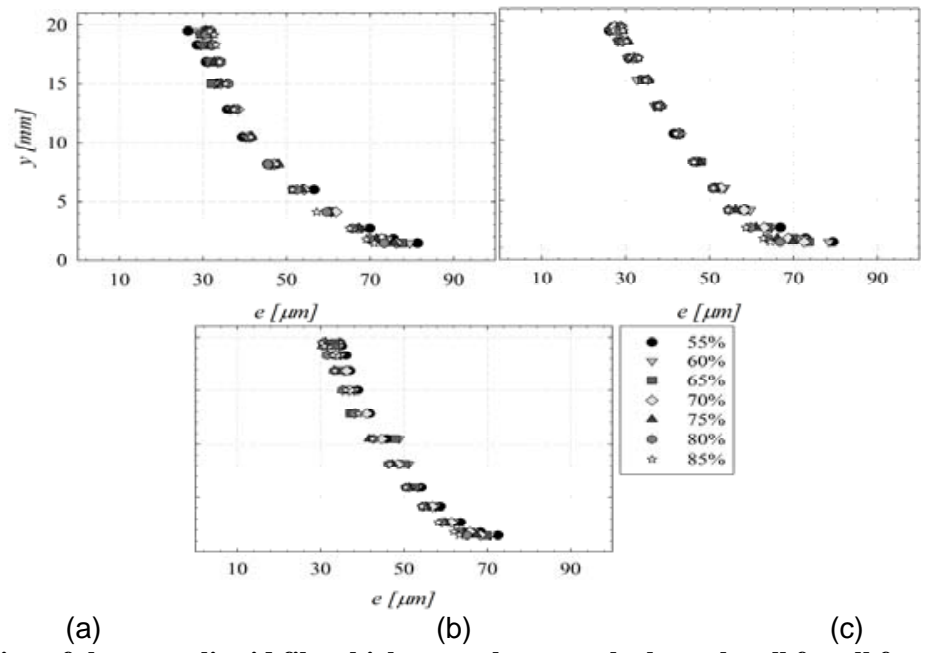

Fig. 13. Evolution of the mean liquid film thickness value over the lateral wall for all foam qualities and the three cases:

(a) Case I, (b) Case II and (c) Case III 


\subsection{Wall shear stress}

One notable aspect of the foam flow is its capacity to generate wall shear stress values 2000 times higher than the water under the same regime. There are many ways to measure the wall shear stress of a fluid inside a channel. It depends of the velocity gradient inside the viscous boundary layer and the pressure losses along a characteristic length. The pressure outlets located along the channel allow obtaining an accurate value of the pressure losses generated by the wall shear stress.

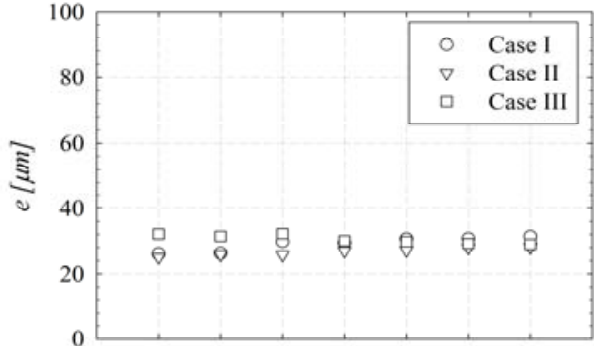

$\begin{array}{lllllll}55 \% & 60 \% & 65 \% & 70 \% & 75 \% & 80 \% & 85 \%\end{array}$

Foam Quality

Fig. 15. Mean liquid film thickness at the bottom of the channel for all cases and qualities.

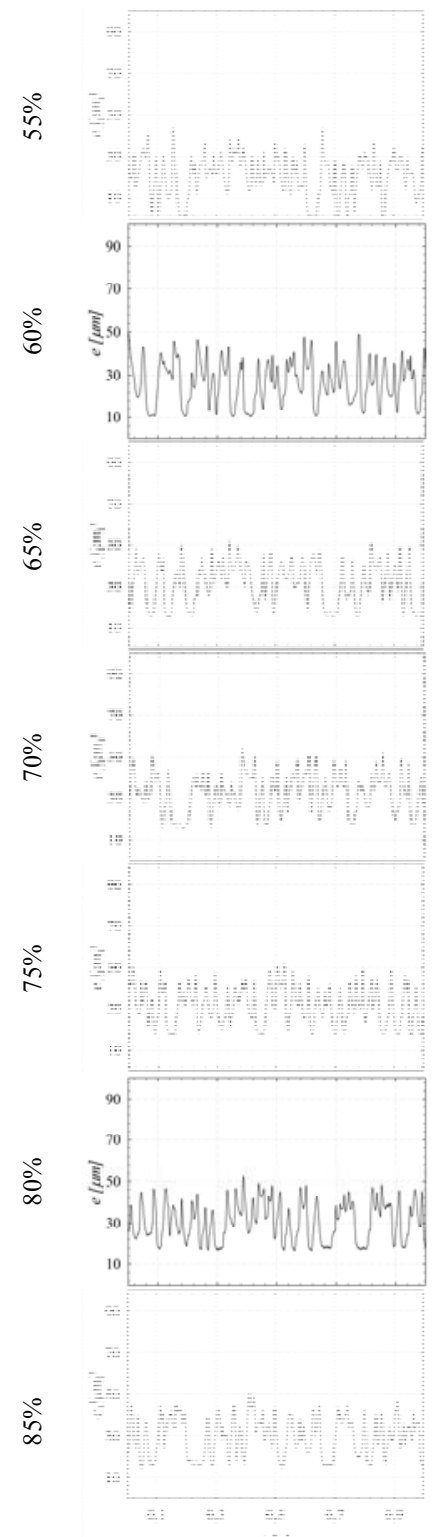

(a)

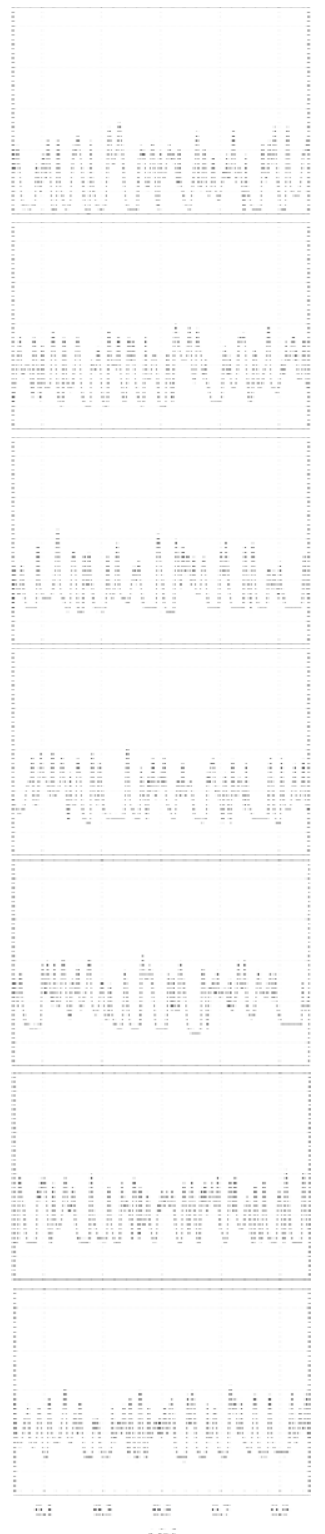

(b)

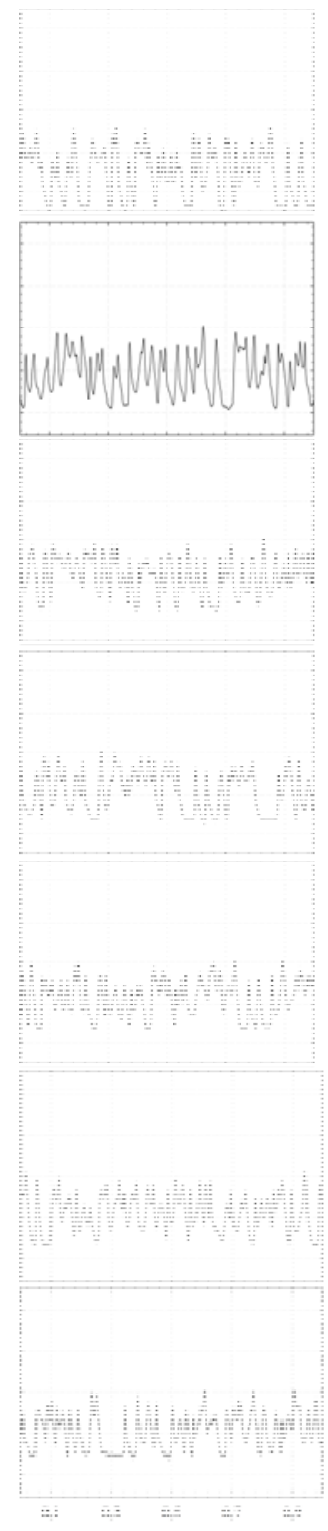

(c)

Fig. 14. Conductimetry signal over the top wall for the Case I at:




The wall shear stress can be obtained through:

$\tau(y)=\mu_{l} \frac{\partial u}{\partial y}$

where $\mu_{l}$ is the liquid's dynamic viscosity and $\frac{\partial u}{\partial y}$ is the velocity gradient.

Foam flow presents complex properties that difficult defining the wall shear stress and the velocity gradient (opacity, reflections, small scales, etc.). The most reliable way to define the mean wall shear stress, for horizontal flowing foam along the channel's surface, is through the pressure losses (Chovet and Aloui 2014; Tisné et al., 2003). They allowed obtaining the pressure gradient along the length of the channel

Due the apparent viscosity of the foam (or consitance of the foam), the pressure gradient for our different cases and qualities allow to deduce the mean wall shear stress using the following relationship:

$\bar{\tau}=\frac{\Delta P}{L} \frac{d_{h}}{4}$

where $\frac{\Delta P}{L}$ is the pressure gradient and $d_{h}=21 \mathrm{~mm}$ is the used hydraulic diameter.

The wall shear stress values obtained with the pressure losses are shown in Fig 16 for all foam qualities and velocities. They show a linear behavior for the Case I and an influence of the quality as the foam velocity increases, especially for the case III. Faster and wetter foams generate higher wall shear stresses.

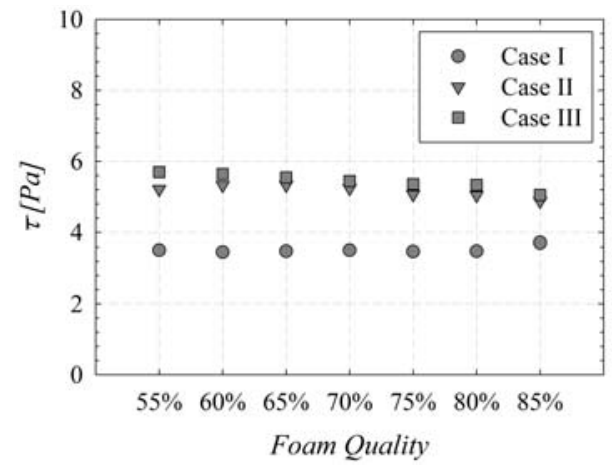

Fig. 16. Mean wall shear stress over the top, lateral and bottom wall of the channel for al qualities and velocities.

\section{CONCLUSIONS}

This experimental study puts into evidence the influence that the liquid slip-layer thickness of flowing foams have over the pressure losses, the velocity fields and the wall shear stress. The measurements of the static pressure, the velocity profiles, and the liquid film thickness allowed us to understand the hydrodynamic behavior of the foam flowing through a straight channel for three different velocity and seven foam qualities. From pressure outlets located all along the channel's length, we defined the pressure losses. They are directly related to the foam velocity and are slightly influenced by the foam quality. Higher velocity generates higher pressure gradient. Using the Particle Imaging Velocimetry (PIV) we were able to determine the local axial velocity of the foam over the periphery of the flow at the lateral wall. They allowed us to verify the three foam regimes, which are influenced near the bottom of the channel by the foam quality and the liquid film thickness. This last one is obtained with the use of conductimetry probes. It evolution over the lateral wall puts into evidence the gravitational drainage of the foam. The top liquid slip-layer thickness remains uniform along the wall. Despite the difference in liquid quantity for the different cases and qualities, thickness remains constant through all fabrication conditions. The only important influence of the foam quality over the slip-layer is at the bottom of the channel, were wetter foams present thicker films. Finally, the wall shear stress results show the small influence that the foam quality and liquid film thicknesses have over the mean value.

\section{ACKNOWLEDGEMENTS}

This experimental work was supported by the LAMIH CNRS UMR 8201 (Department of Mechanics) at the University of Valenciennes (France) and the European Commission within the International Research Staff Exchange Scheme (IRSES) in the $7^{\text {th }}$ Framework Program FP7/2014-2017 under REA grant agreement $\mathrm{n}^{\circ} 612230$. These supports are gratefully acknowledged.

\section{REFERENCES}

Aloui, F. and S. Madani (2007). Wet foam flow under a fence located in the middel of a horizontal duct of square section, Colloid and Surface. A, 309. 71-86.

Aloui, F. and S. Madani. (2008). Experimental investigation of a wetfoam flow through a horizontal sudden expansion, Experimental Thermal and Fluid Science, 32, 905-926.

Blondin, E. and L. Doubliez (2002). Particle imaging velocimetry of a wet aqueous foam with an underlying liquid film, Experiments in Fluids. 32, 294-301.

Chovet, R., L. Keirsbulck and F. Aloui. (2014). Gas-liquid foam through a straight duct and singularities: CFD simulations and experimpents, Proceedings of the ASME, FEDSM2014-21190, pp. V01BT14A003; 8.

Chovet, R., F. Aloui and L. Labraga. (2013). Wet foam flow in horizontal square ducts and through singularities, Proceedings of the ASME, FEDSM2013-16307, pp. 
R. Chovet et al. / JAFM, Vol. 9, Special Issue 1, pp. 39-48, 2016.

\section{V01CT17A012; 7 .}

Chovet, R. F. Aloui and L Keirsbulck (2015). Wall Shear Stress Generated by Aqueous Flowing Foam, Proceedings of the ASME. 57212; Volume 1: Symposia, V001T14A001, AJK Fluids 2015-14039,

Denkov, N. D. S. S. Tcholakova., R Höhler. and S Cohen-Addad. (2012). Foam Rheology, in Foam Engineering: Fundamentals and Applications, John Wiley \& Sons, 91-120.

Durian, D. J. D. A Weitz. and D. J. Pine. (1990). Dynamics and coarsening in three-dimensional foams, J. Phys.: Condensed Matter 2, 433-436.

Hutzler, S. M. Saadatfar., A. Van der Net., D .Weaire. and S. J. Cox (2008). The dynamics of a topological change in a system of soap films,
Coll. Surf. A., 323, 123-131.

Plateau, J. A. F. (1861). 5th and 6th Series, Mem. Acad. Roy. Sci. Belg., 33.

Stevenson, P. (2012). Foam Engineering: Fundamentals and Applications, John Wiley \& Sons, Auckland, New Zealand.

Tisné, P. F. Aloui. and L. Doubliez. (2003). Analysis of wall shear stress in wet foam flows using the electrochemical method, Int. J. Multiphase Flow, 29, 841-854.

Tisné, P. L. Doubliez. and F. Aloui. (2004). Determination of the slip layer thickness for a wet foam flow, Colloids Surf. A: Physicochem. Eng. Aspects, 246, 21-29.

Weaire, D. and S. Hutzler. (1999). The Physics of Foams, Oxford, Dublin. 